\title{
ATLAS Inner Tracker Performance at the beginning of LHC Run 2
}

\author{
Ewa Stanecka \\ INP PAS \\ 9 January 2016
}




\section{Outline}

(1) Physics requirements for precision tracking and vertexing

(2) The Inner Detector overview

(3) Tracking challenge in Run 2

(4) Inner detector upgrade during LS1

- Pixel Detector and IBL

- The Semiconductor Tracker

- The Transition Radiation Tracker

(5) Inner Detector Performance in Run 2

- ID active coverage in 2015

- Pixel Detector and IBL

- SCT Performance

6 Combined tracking performance

- ID material studies

- ID Alignment

- Tracking and vertexing

(7) Summary and outlook 


\section{Physics requirements for precision tracking and vertexing}

Most of the physics analysis in ATLAS require precise measurement of the trajectories of charged particles emerging from LHC collisions as well as precise reconstruction of secondary vertices.

- Precision tracking at LHC luminosity over 5 units in pseudorapidity: $|\eta|<2.5$

- Precise primary/secondary vertex reconstruction

- Excellent b-tagging in jets

- Electron, muon, tau, b- and c-hadron reconstruction

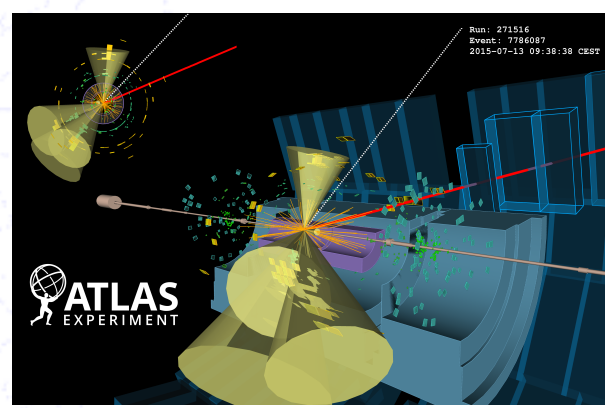




\section{The Inner Detector overview}

Immersed in $2 T$ solenoid magnetic field:

- $80 \mathrm{M}$ channel Pixel Detector, resolution $10 \times 115 \mu \mathrm{m}+$ new IBL 4th Pixel layer with planar \& 3D silicon sensors, resolution $8 \times 40 \mu \mathrm{m}$

- $6 \mathrm{M}$ channel Silicon microstrips (SCT), resolution $17 \times 580 \mu \mathrm{m}$

- 350k ch. Transition Radiation Tracker (TRT), resolution $\sim 130 \mu \mathrm{m}$
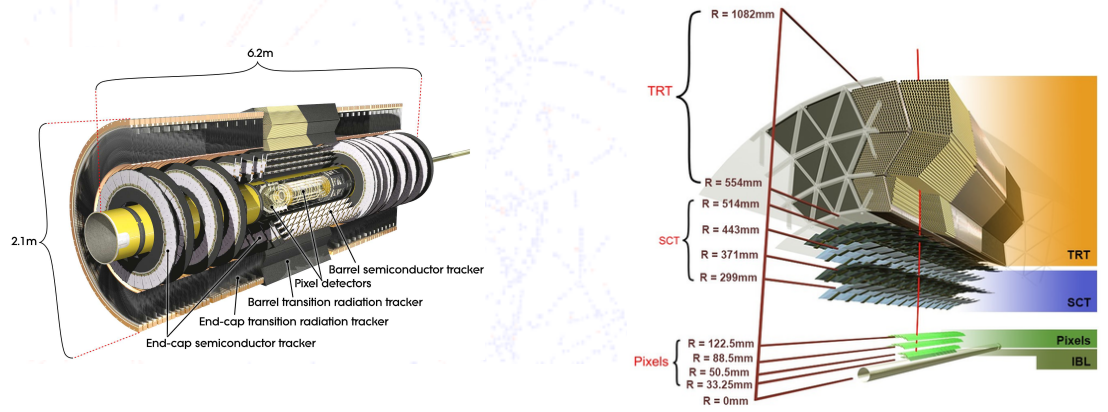


\section{ATLAS tracking challenge in Run 2}

The LHC underwent several upgrades during the long shutdown period: beam energy $7 \mathrm{TeV} \rightarrow 13 \mathrm{TeV}$, bunch crossing 50 ns $\rightarrow 25$ ns, luminosity increase $\Rightarrow$ stress on detector hardware and software:

- High pile up - in Run 1 up to 40 $p p$ interactions per bunch crossing.

- High detector occupancy.

- Non-zero rate of Single Event Upsets (SEUs)

- Optimize pattern finding and reconstruction algorithms for dense environment.

- Improvement in computing models to handle trigger rate and large event size

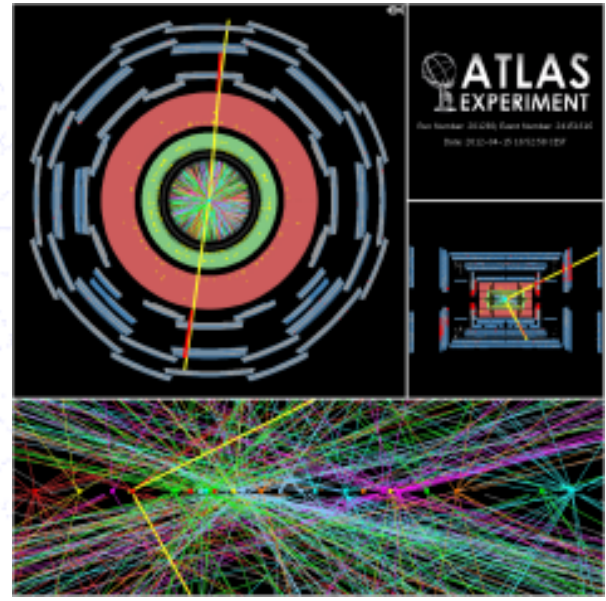




\section{Inner detector upgrade during LS1: Pixel and IBL}

The Pixel Detector was extracted and serviced on surface:

- Repaired all accessible failures

- On-detector services replaced and opto electronics moved to off-detector location for improved accessibility

- New optical links installed with an increase data bandwidth capability for Run 2 for L1/L2 \& beyond $\left(3 \times 10^{34} \mathrm{~cm}^{-2} \mathrm{~s}^{-1}\right)$

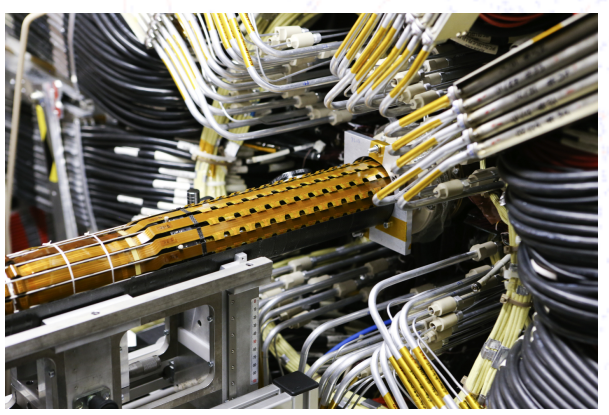

New insertable B-layer (IBL) :

- additional pixel layer at $33 \mathrm{~mm}$ from beam line

- IBL assures better track and vertex reconstruction performance at high luminosity expected during run 2 and mitigates the impact of radiation damage to the B-layer. 


\section{Inner detector upgrade during LS1: SCT}

- Upgrade/expansion of SCT

DAQ

- Installation of an additional 38 Read-Out Drivers (RODs) to remove a critical DAQ bottleneck, allow to read out the SCT up to $3 \times 10^{34} \mathrm{~cm}^{-2} \mathrm{~s}^{-1}$ (at 25ns bunch spacing)

- Installation of new TX optical engines in the Back Of Crate

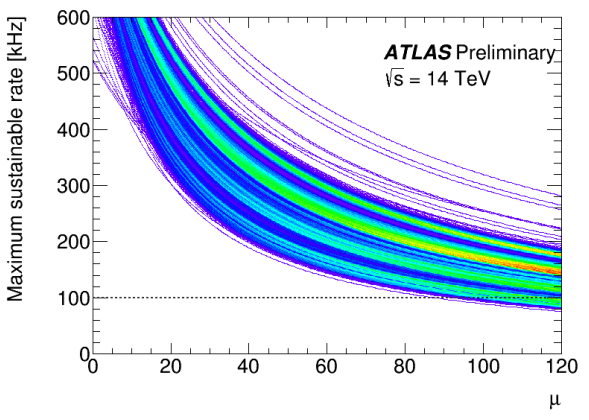
(BOC) cards.

- ROD firmware upgrade

- Auto recovery of modules with non-zero errors.

- Periodic reconfiguration to protect against the spontaneous corruption of the configuration (single event upset).

- Stop-less reconfiguration \& reintegration of RODs in case of busy. 


\section{Inner detector upgrade during LS1: TRT}

- Acive gas issues:

- Leaks of Xenon developed at the end of Run1

- Leaks in end-cap successfully repaired

- DCS system for leak control implemented

- Operation scenarios prepared: parts of TRT will run with Ar-mixture

- Performance was tested and it was demonstrated that the tracking properties are not changed.

- TR performance is still supported with other parts of the TRT running with Xe mixture

- DAQ enhancement for high intensity and high occupancy running.

- HV PS improvements to provide protection against HV overshoot at the beam dump.

- PID improvements for high luminosity/occupancy 


\section{Inner Detector active coverage}

\begin{tabular}{|l|c|c|}
\hline Subdetector & Number of Channels & Approximate Operational Fraction \\
\hline Pixels & $92 \mathrm{M}$ & $98.2 \%$ \\
\hline SCT Silicon Strips & $6.3 \mathrm{M}$ & $98.6 \%$ \\
\hline TRT Transition Radiation Tracker & $350 \mathrm{k}$ & $97.3 \%$ \\
\hline
\end{tabular}

- For the Pixel status:

- 3-Layers Pixel (80 M channels) - 98\%

- IBL (12 M channels) - 99.5\%

- SCT expected to rise to $98.9 \%$ after including temporarily disabled modules. 


\section{Pixel and IBL Performance - Detector Timing}

- Time-over-Threshold (ToT, length of discriminator signal); depends on deposited charge, discriminator threshold and feedback current.

- Pixel Detector re-timed in during early 50 ns runs

- Hits with ToT $\leq 7$ recovered by "hit duplication" mechanism from next BC

- IBL Maintained nearly 100\% efficiency for 1 BC readout

- For the 25 ns run, hits with TOT $\leq 2$ (suffering from time-walk) recovered thanks to FE-I4 special functionalities (HitDiscCnfg).
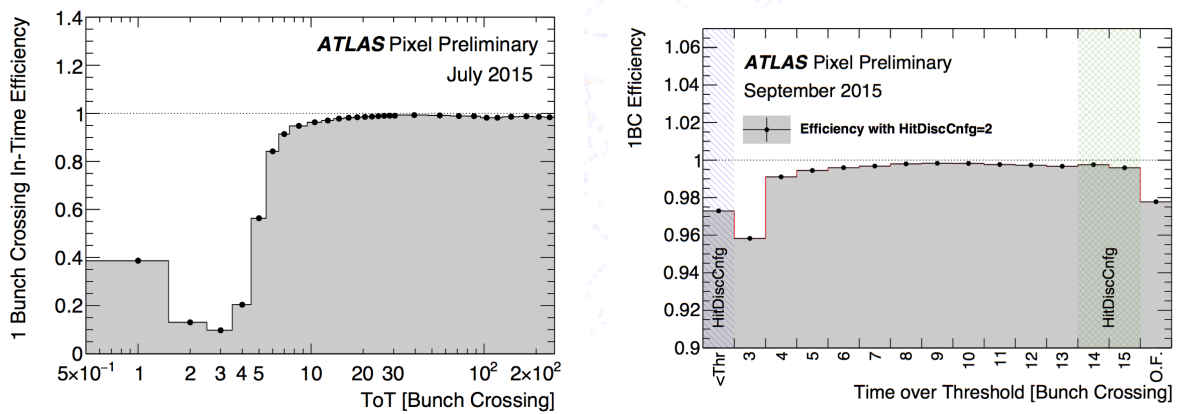

Ewa Stanecka (INP PAS)

ATLAS Inner Tracker Performance

9 January 2016

$10 / 18$ 


\section{SCT Performance - calibration}

- Noise comparison between end of Run 1 and pre-Run 2 data.

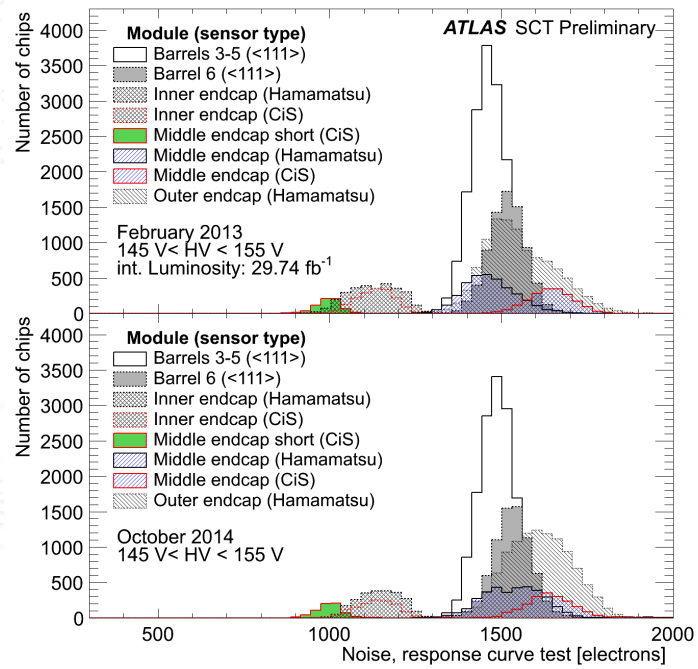




\section{SCT Performance at $25 \mathrm{~ns}$}

All SCT performance metrics are comparable to Run1, except for the impact on hit efficiencies with $25 \mathrm{~ns}$.

- SCT reads-out three time bins per strip: previous $B C$, current $B C$, next BC (XXX)

- Online reads-out via $\mathrm{X} 1 \mathrm{X}$; offline require $01 \mathrm{X}$ to reject ghost tracks from previous BC

- but strips, that are hit again in the current $\mathrm{BC}$ are lost $\Rightarrow 0,5 \%$ hit efficiency drop

The intrinsic SCT hit efficiency can be determined using the first bunch in the train.

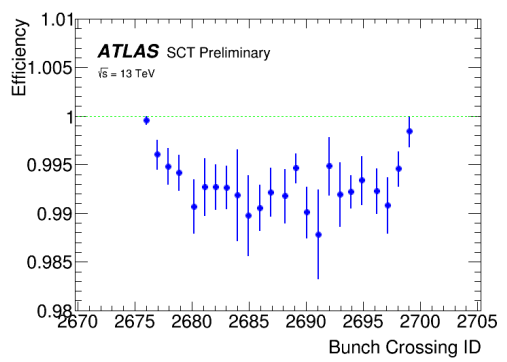




\section{Combined performance - ID material studies}

- IBL, new Beam Pipe and Services $\Rightarrow$ additional material.

- Material studies using hadronic interactions and conversions have led to a new geometry for simulations.
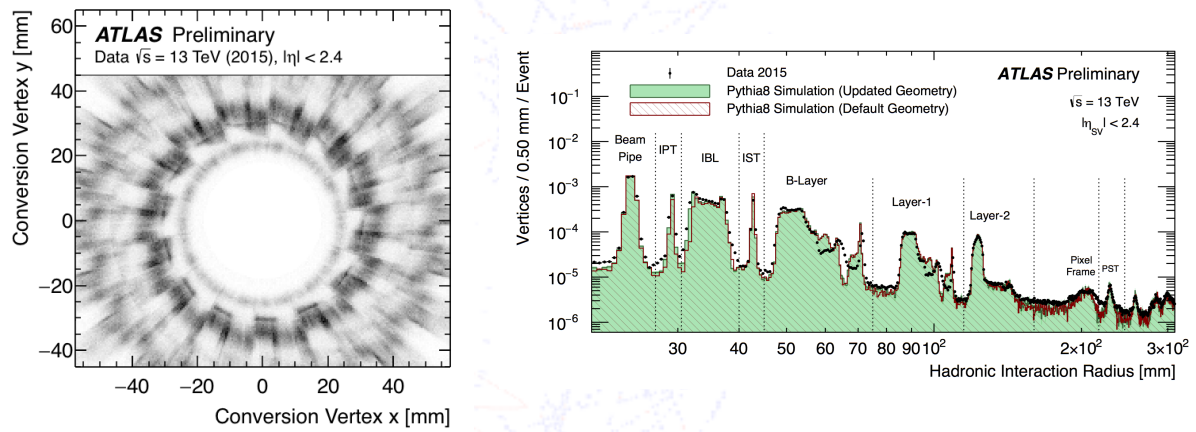


\section{Combined performance -Alignment}

- $13 \mathrm{TeV}$ pp collisions used to align Inner Detector with a track based algorithm. ID stable at a $\mu \mathrm{m}$ level under regular operational condition.

- Special focus on the IBL detector - observed distortion related to $\triangle T$

- Alignment corrections for fill and lumi blocks mitigate the effect of of the IBL distortion.
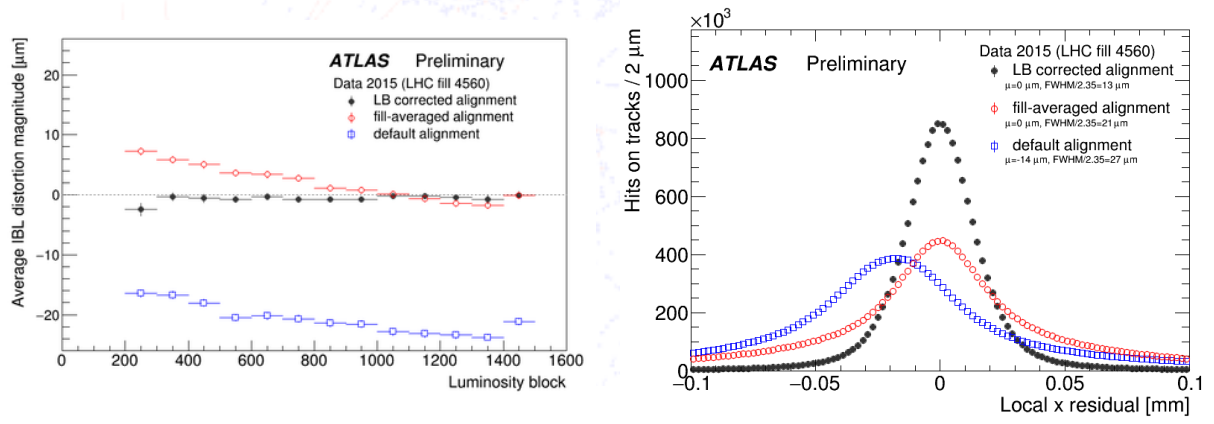


\section{Combined performance - Track impact parameter}

The impact parameter resolution improved with IBL.
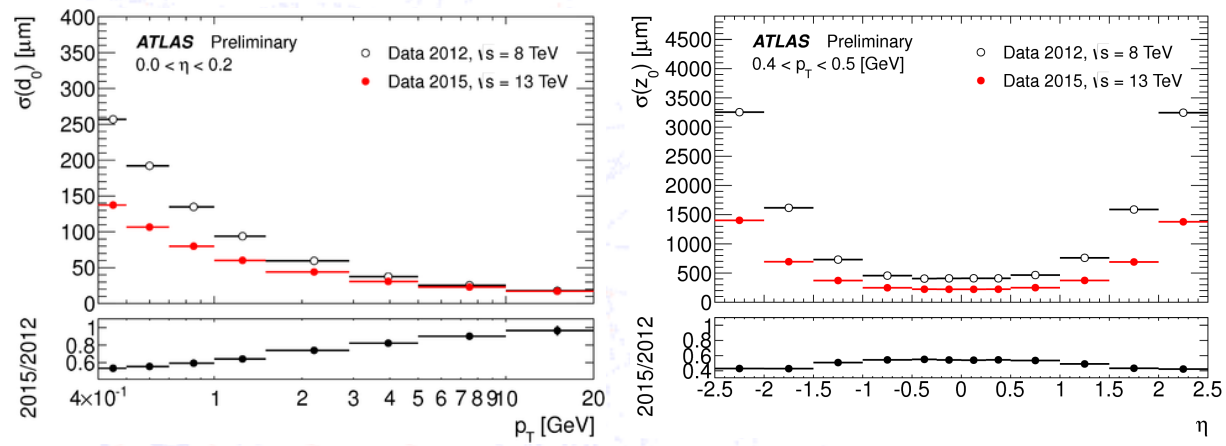


\section{Combined performance - Vertex reconstruction}

- Vertex reconstruction performance studied in $13 \mathrm{TeV}$ data with lowand high- number of $p p$ interactions per bunch crossing $(\mu)$.

- Vertex resolution estimated using Split Vertices method
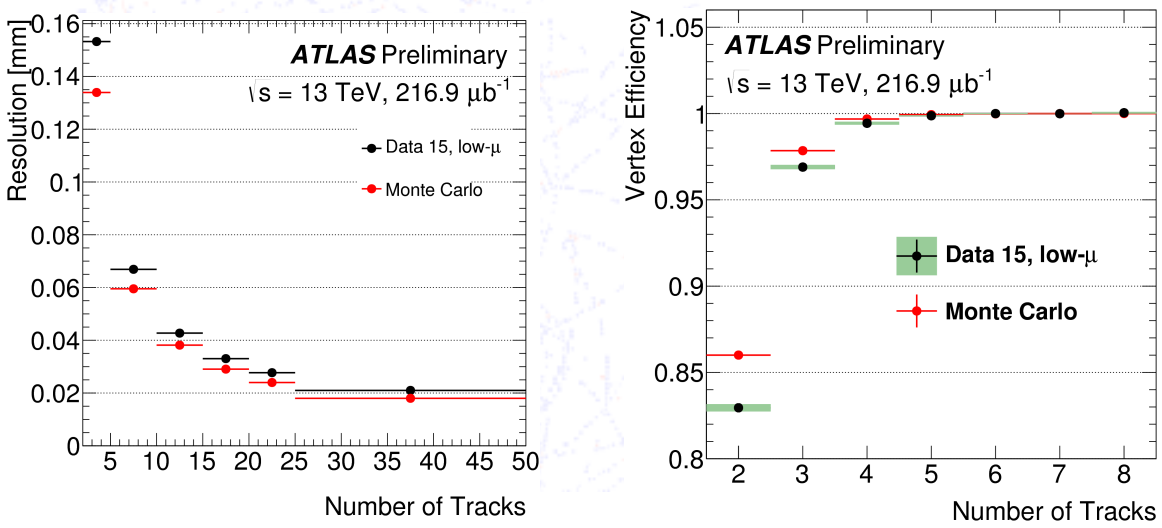


\section{Summary and outlook}

- TRT, SCT and Pixel Detector + IBL (re)commissioned after LS1 upgrades with $13 \mathrm{TeV}$ data

- All subsystems proved to work reliably with $13 \mathrm{TeV}$ collissions at 25 ns bunch crossing

- Combined Inner Detector tracking performance improved with respect to Run-1 thanks to IBL.

- Looking forward to more data in 2016! 


\section{Thank you for your attention!}

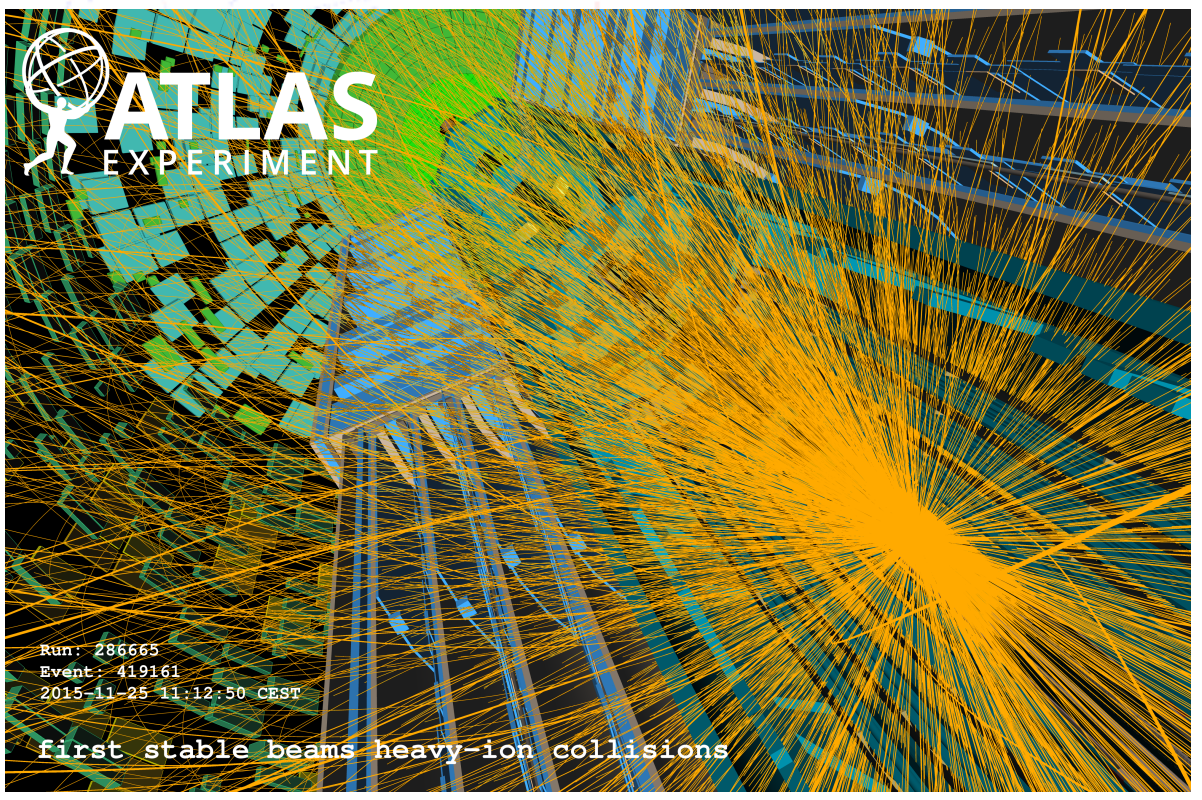

